\title{
The Use of Alveolar Dead Space Fraction to Predict Postoperative Outcomes after Pediatric Cardiac Surgery: A Retrospective Study
}

\author{
Imran A. Sayed ${ }^{1} \cdot$ Scott Hagen ${ }^{2} \cdot$ Victoria Rajamanickam $^{3} \cdot$ Petros V. Anagnostopoulos ${ }^{4} \cdot$ Marlowe Eldridge $^{2}$. \\ Awni Al-Subu² $\mathbb{}$
}

Received: 4 February 2021 / Accepted: 30 June 2021 / Published online: 9 July 2021

(c) The Author(s), under exclusive licence to Springer Science+Business Media, LLC, part of Springer Nature 2021

\begin{abstract}
Patients with congenital heart disease (CHD) that have surgical repair with cardiopulmonary bypass (CPB) reflect a unique population with multiple pulmonary and systemic factors that may contribute to increased alveolar dead space and low cardiac output syndrome. This study aimed to assess and compare changes in the alveolar dead space fraction (AVDSf) in the immediate postoperative period with outcomes in children with CHD who underwent repair on CPB. A single-center retrospective review study of critically ill children with CHD, younger than 18 years of age admitted to the Pediatric Intensive Care Unit (PICU) after undergoing surgical repair on CPB and received invasive mechanical ventilation for at least $24 \mathrm{~h}$. One hundred and two patients were included in the study. Over the first $24 \mathrm{~h}$, mean AVDSf was significantly higher in patients who had longer hospital length of stay (LOS) ( $>21$ days) $p=0.02$, and longer duration of invasive mechanical ventilation (DMV) $(>170 \mathrm{~h}) p=0.01$. Cross-sectional analyses at $23-24$ h revealed that AVDSf $>0.25$ predicts mortality and DMV ( $p=0.03$ and $\mathrm{P}=0.02$ respectively); however, it did not predict prolonged hospital LOS. For every 0.1 increase in the AVDSf, the odds of mortality, DMV, and hospital LOS increased by 4.9 [95\% CI $=1.45-16.60, p=0.002], 2.06$ [95\% $\mathrm{CI}=1.14-3.71, p=0.01]$, and $1.43[95 \% \mathrm{CI}=0.84-2.45, p=0.184]$, respectively. The area under the ROC curve at 23-24 $\mathrm{h}$ for AVDSf was 0.868 to predict mortality as an outcome. AVDSf $>0.25$ at $23-24 \mathrm{~h}$ postoperatively was an independent predictor of mortality with sensitivity and specificity of $83 \%$ and $80 \%$, respectively and was superior to other commonly used surrogates of cardiac output. In the immediate postoperative period of pediatric patients with CHD, high AVDSf is associated with longer hospital length of stay and duration of invasive mechanical ventilation. Increased AVDSf values at 23-24 h postoperatively is associated with mortality in patients with CHD exposed to CPB.
\end{abstract}

Keywords Regional oxygen saturation $\cdot$ Alveolar dead space fraction $\cdot$ Non-invasive $\cdot$ Monitoring $\cdot$ Pediatrics $\cdot$ Congenital heart disease

Awni Al-Subu

al-subu@pediatrics.wisc.edu

1 Department of Pediatrics, University of Colorado Anschutz Medical Campus, Children's Hospital of Colorado, Colorado Springs, CO, USA

2 Division of Pediatric Critical Care Medicine, Department of Pediatrics, University of Wisconsin, 600 Highland Ave, Room H6/535 CSC, Madison, WI 53792, USA

3 Division of Biostatistics, Department of Biostatistics and Medical Informatics, University of Wisconsin, Madison, WI, USA

4 Division of Pediatric Cardiothoracic Surgery, Department of Surgery, University of Wisconsin, Madison, WI, USA

$\begin{array}{ll}\text { Abbreviations } \\ \text { AVDSf } & \text { Alveolar dead space fraction } \\ \mathrm{CHD} & \text { Congenital heart disease } \\ \mathrm{CPB} & \text { Cardiopulmonary bypass } \\ \mathrm{DMV} & \text { Duration of invasive mechanical ventilation } \\ \mathrm{EMR} & \text { Electronic medical record } \\ \mathrm{EtCO}_{2} & \text { End-tidal carbon dioxide } \\ \mathrm{IQR} & \text { Interquartile range } \\ \mathrm{IRB} & \text { Institutional Review Board } \\ \mathrm{LOS} & \text { Length of stay } \\ \mathrm{MAP} & \text { Mean arterial blood pressure } \\ \mathrm{NIRS} & \text { Near-Infrared Spectroscopy and Imaging } \\ \mathrm{PaCO} & \text { Partial pressure of carbon dioxide } \\ \mathrm{PICU} & \text { Pediatric intensive care unit } \\ \mathrm{ROC}^{2} & \text { Receiver operating characteristic } \\ \mathrm{rSO}_{2} & \text { Regional oxygen saturations }\end{array}$

Abbreviations

AVDSf Alveolar dead space fraction

CHD Congenital heart disease

CPB Cardiopulmonary bypass

DMV Duration of invasive mechanical ventilation

EMR Electronic medical record

$\mathrm{EtCO}_{2}$ End-tidal carbon dioxide

IQR Interquartile range

IRB Institutional Review Board

LOS Length of stay

MAP Mean arterial blood pressure

NIRS Near-Infrared Spectroscopy and Imaging

$\mathrm{PaCO}_{2} \quad$ Partial pressure of carbon dioxide

PICU Pediatric intensive care unit

$\mathrm{rSO}_{2} \quad$ Regional oxygen saturations 
STS Society of Thoracic Surgeons

V/Q Ventilation-perfusion

VPS Virtual PICU Systems

\section{Introduction}

Patients with congenital heart disease (CHD) that have surgical repair with cardiopulmonary bypass (CPB) reflect a unique population with multiple pulmonary and systemic factors that may contribute to increased ventilation-perfusion (V/Q) mismatch and low cardiac output syndrome (LCOS). Both V/Q mismatch and LCOS are associated with prolonged mechanical ventilation, ICU length of stay, hospital length of stay (LOS), and increased cost [1-2]. Traditionally, invasive monitoring has been used to assess cardiac output and adequacy of ventilation, but invasive monitoring has the potential for substantial risks and complications [3, 4].

In an attempt to minimize the risk of such complications, non-invasive monitoring of alveolar dead space-to-alveolar tidal volume $(\mathrm{Vd} / \mathrm{Vt})$, also known the pulmonary dead space fraction, using capnography are increasingly used to guide postoperative management of critically ill children with CHD [5]. Pulmonary dead space is the portion of inhaled air in the alveoli that does not participate in gas exchange and quantifies the magnitude of ventilation-perfusion abnormalities in the lung [5, 6]. Pulmonary dead space fraction $(\mathrm{Vd} /$ $\mathrm{Vt}$ ) can be easily measured in patients receiving mechanical ventilation using bedside volumetric capnometry and $\mathrm{PaCO}_{2}$ values [7]. $\mathrm{Vd} / \mathrm{Vt}$ has been shown in adult and pediatric populations to have significant prognostic value in acute lung injury [8-10].

Infants and children with CHD have been shown to have abnormal Vd/Vt. Moreover, recent studies have shown that higher $\mathrm{Vd} / \mathrm{Vt}$ is associated with outcomes such as duration of mechanical ventilation and hospitalization following cardiac surgery $[5,11]$. However, $\mathrm{Vd} / \mathrm{Vt}$ measurement requires the use of volumetric capnography and can be quite burdensome and is not routinely monitored in the intensive care unit [12]. Alveolar dead space fraction (AVDSf), which is easily estimated using standard time-based capnography and arterial blood gas analysis, can be used as a reasonable surrogate for $\mathrm{Vd} / \mathrm{Vt}$ [13]. Since most mechanically ventilated children are monitored using time-based capnography, AVDSf has been more commonly used to easily and simply estimate $\mathrm{Vd} /$ $\mathrm{Vt}$ at the bedside [2,14-16]. AVDSf has been shown in both pediatric and adult patient populations to have an important prognostic value in acute lung injury $[16,17,18]$. However, given that increased dead space in patients with CHD has multiple etiologies and not only the result of intrapulmonary shunt or the acute lung injury in response to $\mathrm{CPB}$, the use of AVDSf in intubated infants and children with CHD in the immediate postoperative period is limited [17].
In this study we postulate that using AVDSf cut-off values may facilitate early recognition and early intervention, which could possibly help to alleviate an evolving problem. This study aimed to assess and compare AVDSf measured during the first 24 postoperative hours with outcomes in children with CHD who underwent repair on CPB. Our primary outcome was hospital mortality. Our secondary outcomes were hospital LOS greater than 21 days and duration of invasive mechanical ventilation (DMV) greater than $170 \mathrm{~h}$.

\section{Methods}

This was a single-center retrospective study. The Institutional Review Board (IRB) at the University of Wisconsin, Madison approved the study protocol with a waiver of informed consent.

\section{Study Population}

A retrospective chart review was conducted for all pediatric patients $\leq 18$ years of age who underwent repair of CHD between January 2012 and August 2016 at the American Family Children's Hospital. We included only the patients who were repaired using $\mathrm{CPB}$, intubated, with cuffed endotracheal tube, and mechanically ventilated for at least $24 \mathrm{~h}$ after PICU admission with an arterial catheter in place. We excluded patients placed on extracorporeal membrane oxygenation intraoperatively or those extubated within 24 postoperative hours. All patients were monitored with an end-tidal carbon dioxide $\left(\mathrm{EtCO}_{2}\right)$ capnostat (Capnostream $\mathrm{CO}_{2}$ monitors, Medtronic/Covidien, Minneapolis, $\mathrm{MN}$, USA) placed between the endotracheal tube and ventilator circuit. $\mathrm{EtCO}_{2}$ values were recorded hourly as per PICU protocol in the electronic medical records (EMR).

\section{Data Collection}

The medical records of patients who met the inclusion criteria were reviewed. Perioperative and intraoperative data collected included demographics, diagnosis, standard hemodynamic and respiratory monitoring data, commonly used surrogates of cardiac output [arterial blood gases, serum lactate, mean arterial blood pressure (MAP), cerebral and renal regional oxygen saturations $\left(\mathrm{rSO}_{2}\right)$ obtained via NearInfrared Spectroscopy (NIRS)], total CPB time, cross-clamp time, circulatory arrest time, shunt type, shunt size, ventilator settings for the first $24 \mathrm{~h}$, duration of mechanical ventilation, PICU and hospital LOS, and survival rates. All the above data were obtained by abstraction from EMR, Virtual PICU Systems (VPS) database, and Society of Thoracic Surgeons (STS) database. 
We obtained the partial pressure of carbon dioxide $\left(\mathrm{PaCO}_{2}\right)$ values from arterial blood gas measurements for the first $24 \mathrm{~h}$. Corresponding $\mathrm{EtCO}_{2}$ values were collected from the EMR. Calculation of AVDSf was performed using the following equation: $\mathrm{AVDSf}=\left(\mathrm{PaCO}_{2}-\mathrm{EtCO}_{2}\right) / \mathrm{PaCO}_{2}$ [19].

Our primary outcome measure was postoperative mortality. Based on previous studies and reports, the secondary outcomes were defined as DMV $>170 \mathrm{~h}$ and LOS longer than 21 days.

\section{Statistical Methodology}

Association of demographic and intraoperative categorical variables with primary and secondary outcomes such as hospital mortality, LOS $>21$ days vs. $\leq 21$ days, and DMV $>170 \mathrm{~h}$ vs. $\leq 170 \mathrm{~h}$ was analyzed using the Fisher's exact and Wilcoxon rank-sum tests as appropriate.

Calculated AVDSf was aggregated in 2-h time intervals. To assess the trend over time of AVDSf between the primary and secondary outcomes, a polynomial mixed-effect model is used. Given that shunt fraction can have significant clinical importance on AVDSf, additional subgroup analysis, based on physiology and presence of shunt, 2 ventricle physiology with no shunt, 2 ventricle physiology with intracardiac shunt, and single ventricle was performed. Finally, we also performed additional analysis with traditional bedside surrogates of cardiac input including serum lactate, $\mathrm{pH}$, and changes in blood pressure using the same method as above for AVDSf.

The AVDSf was log-transformed to ensure linearity, and the time variable was considered continuous in the model. For the trend analysis of AVDSf, a mixed-effect model with a polynomial of degree 3 was chosen based on LRT (likelihood ratio test). Predicted means were generated for each time point and back transformed to represent geometric means. Contrast statement was used to access the joint distribution of main and interaction effects to determine whether the trend was significantly different. The effect of outcomes at specific time intervals was evaluated using estimate statements in the models.

Logistic regression models were fit to determine the OR for one standard deviation increase in AVDSf in predicting the primary and secondary outcomes at 23-24 h' time interval. Receiver operating characteristic (ROC) analyses were conducted to assess the optimal cut-off values for prediction. A $p$ value $<0.05$ was considered statistically significant. SAS software (version 9.4, SAS Institute, Cary, NC) was used for all statistical analyses.

\section{Results}

Total of 315 patients underwent cardiac surgery with CBP and received invasive mechanical ventilation for at least $24 \mathrm{~h}$ during the 5-year study period. One hundred and two patients were included in the study (Fig. 1). All demographic data, perioperative risk factors, invasive markers of cardiac output, and outcomes are presented in Table 1. Median (IQR) age and weight at PICU admission were $0.1(0.00-3.3)$ months and $3.7(3.15-5.42)$ Kilograms, respectively. Overall, 63 patients (61.2\%) were male and 14 (13.5\%) had single ventricle physiology. Hospital mortality was associated with lower gestational age and race. Nonsurvivors had longer DMV 1084.00 (342.0-2089.0) hours, $p<0.001$ and longer hospital LOS 54.00 (26.63-96.00) days, $p=0.033$. Median serum lactate, $\mathrm{pH}$, and invasive MAP (as an invasive surrogates of CO) over the first $24 \mathrm{~h}$, were not statistically significant between survivors and nonsurvivors with $p=0.593,0.667$, and 0.927 , respectively (Table 1) (supplementary Fig. S1). Similarly, cerebral and renal $\mathrm{rSO}_{2}$ (as a non-invasive surrogates of $\mathrm{CO}$ ) over the first $24 \mathrm{~h}$ were not statistically significant with $p=0.11$ and 0.2 , respectively (supplementary Fig. S2). Demographics and basic characteristics, serum lactate, invasive MAP, and arterial $\mathrm{pH}$ pertaining to secondary outcomes are illustrated in Supplementary Table S1 and Supplementary Table S2.

Over the first $24 \mathrm{~h}$, mean AVDSf was significantly higher in patients who had longer hospital LOS ( $>21$ days) $p=0.02$ and longer DMV $(>170$ h) $p=0.01$. Although the mean AVDSf over the first $24 \mathrm{~h}$ was higher in patients who did not survive to discharge, that difference was not statistically significant, $p=0.16$ (Fig. 2). Cross-sectional analyses at 23-24 h' time interval revealed that AVDSf higher than 0.25 predicts hospital mortality and DMV $(p=0.03$ and $\mathrm{P}=0.02$, respectively); however, it did not predict prolonged hospital LOS (Fig. 2). For every 0.1 (1 SD) increase in the AVDSf the odds of mortality, DMV, and hospital LOS increased by 4.9, 2.06, and 1.43, respectively (Table 2). Subgroup analysis for AVDSf did not demonstrate a significant

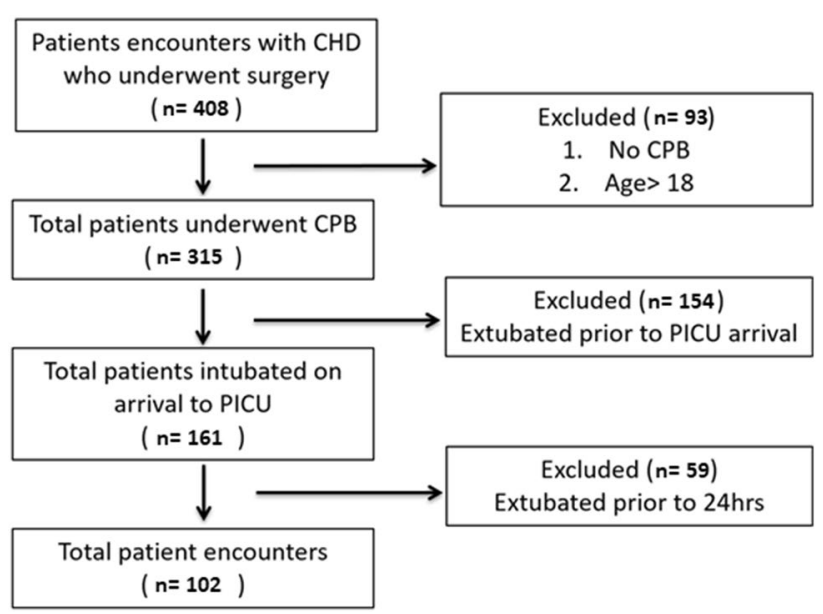

Fig. 1 Consort diagram of the study. CHD: congenital heart Disease, CPB: cardiopulmonary bypass, PICU: pediatric intensive care unit 
Table 1 Demographics and baseline characteristics of all patients and comparison between survivors and non-survivors

\begin{tabular}{|c|c|c|c|c|}
\hline Characteristics & All & Survivors & Non-Survivors & $p$ value* \\
\hline $\mathrm{n}$ & 102 & 93 & 9 & NA \\
\hline \multicolumn{5}{|l|}{ Demographics } \\
\hline Age (months): median (IQR) & $0.1(0.00-3.3)$ & $0.12(0.04-2.67)$ & $0.83(0.04-3.7)$ & 0.674 \\
\hline Gestational age at birth (weeks): median (IQR) & $39.0(38.0-39.0)$ & $39.0(38.0-39.0)$ & $37.0(36.0-38.0)$ & 0.016 \\
\hline Weight (Kilograms): median (IQR) & $3.7(3.15-5.42)$ & $3.67(3.17-5.40)$ & $3.93(2.7-5.65)$ & 0.83 \\
\hline Height (inch): median (IQR) & $21.1(20.07-23.5)$ & $21.06(20.07-23.0)$ & $24.01(22.4-24.8)$ & 0.24 \\
\hline Male, gender, n (\%) & $63(61.17)$ & $57(61.29)$ & $6(66.67)$ & 1.00 \\
\hline Birth weight (Kilograms): median (IQR) & $3.10(2.8-3.43)$ & $3.12(2.87-3.45)$ & $2.57(2.13-2.94)$ & 0.065 \\
\hline Race & & & & 0.002 \\
\hline White $\mathrm{n}(\%)$ & $88(86.27)$ & $84(90.32)$ & $4(44.44)$ & \\
\hline Others n $(\%)$ & $14(13.73)$ & $9(9.68)$ & $5(55.56)$ & \\
\hline Ethnicity & & & & 1.00 \\
\hline Hispanic n (\%) & $11(10.68)$ & $10(10.75)$ & $1(1.11)$ & \\
\hline Not Hispanic n (\%) & $91(89.22)$ & $83(89.25)$ & $8(88.89)$ & \\
\hline \multicolumn{5}{|l|}{ Intraoperative } \\
\hline CPB time (minutes) median (IQR) & $173(131-204)$ & $173(133-203)$ & $108.00(90-214)$ & 0.392 \\
\hline Aortic clamp duration (minutes): median (IQR) & $83(48.2-107)$ & $83(49-107)$ & $64.50(23.5-103.00)$ & 0.485 \\
\hline STAT Category, $n(\%)$ & & & & 0.91 \\
\hline 1 & $5(4.90)$ & $5(5.38)$ & $0(0)$ & \\
\hline 2 & $26(25.49)$ & $23(24.73)$ & $3(33.33)$ & \\
\hline 3 & $21(2.59)$ & $20(21.51)$ & $1(11.11)$ & \\
\hline 4 & $41(40.20)$ & 37 (39.78) & $4(44.44)$ & \\
\hline 5 & $9(8.82)$ & $8(8.60)$ & $1(11.11)$ & \\
\hline Shunts & & & & 0.717 \\
\hline Present & $36(35.29)$ & $32(34.41)$ & $4(44.44)$ & \\
\hline Not present & $66(64.71)$ & $61(65.59)$ & $5(55.56)$ & \\
\hline Single ventricle & $14(13.73)$ & $11(11.83)$ & $3(33.33)$ & 0.105 \\
\hline \multicolumn{5}{|l|}{ Secondary Outcomes } \\
\hline Hospital LOS (day), median (IQR) & $21.8(13.13-36.96)$ & $20.88(12.46-33.17)$ & $54.00(26.63-96.00)$ & 0.033 \\
\hline DMV (hours): median (IQR) & $143.5(78.0-291.0)$ & $128(76-197)$ & $1084.00(342.0-2089.0)$ & $<0.0001$ \\
\hline \multicolumn{5}{|l|}{ Lactate, $\mathrm{pH}$, and invasive $\mathrm{BP}$ over the first $24 \mathrm{~h}$} \\
\hline Lactate: median (IQR) & $3.7(2.1-5.3)$ & $3.8(2.3-5.3)$ & $2.5(1.7-6.8)$ & 0.593 \\
\hline BP: median (IQR) & $56.67(48.6-66.6)$ & $57.00(48.8-66.1)$ & $54.00(47.3-75.0)$ & 0.927 \\
\hline pH: median (IQR) & $7.38(7.3-7.4)$ & $7.38(7.3-7.4)$ & $7.35(7.3-7.4)$ & 0.667 \\
\hline
\end{tabular}

$C P B$ Cardiopulmonary bypass, $D M V$ duration of invasive mechanical ventilation, IQR interquartile range, $L O S$ length of stay, STAT The Society of Thoracic Surgeons-European Association for Cardio-Thoracic Surgery) score

* $p$ value is shown for comparison between patients with postoperative survival vs non-survival

association with mortality (Fig. 3), hospital LOS (Fig. 4), or DMV (Fig. 5) in the patients with no shunts, residual shunts (both right-to-left and left-to-right shunts), or single ventricle physiology.

The area under the ROC curve at 23-24 h for AVDSf was 0.868 to predict mortality as an outcome, which was superior to other commonly used surrogates of CO. Only AVDSf $>0.25$ at $23-24 \mathrm{~h}$ postoperatively was an independent predictor of hospital mortality with sensitivity and specificity of $83 \%$ and $80 \%$, respectively (Fig. 6A). The area under the ROC curve at 23-24 h for AVDSf to predict hospital LOS and DMV are shown in (Fig. 6B, C). The area under the ROC curve at 23-24 h for commonly used invasive and non-invasive surrogates of $\mathrm{CO}$ to predict hospital LOS and DMV are shown in (Fig. 6A-C).

\section{Discussion}

In this retrospective study of critically ill children with $\mathrm{CHD}$ who underwent surgical repair on $\mathrm{CPB}$ and required mechanical ventilation $>24 \mathrm{~h}$ during the postoperative 

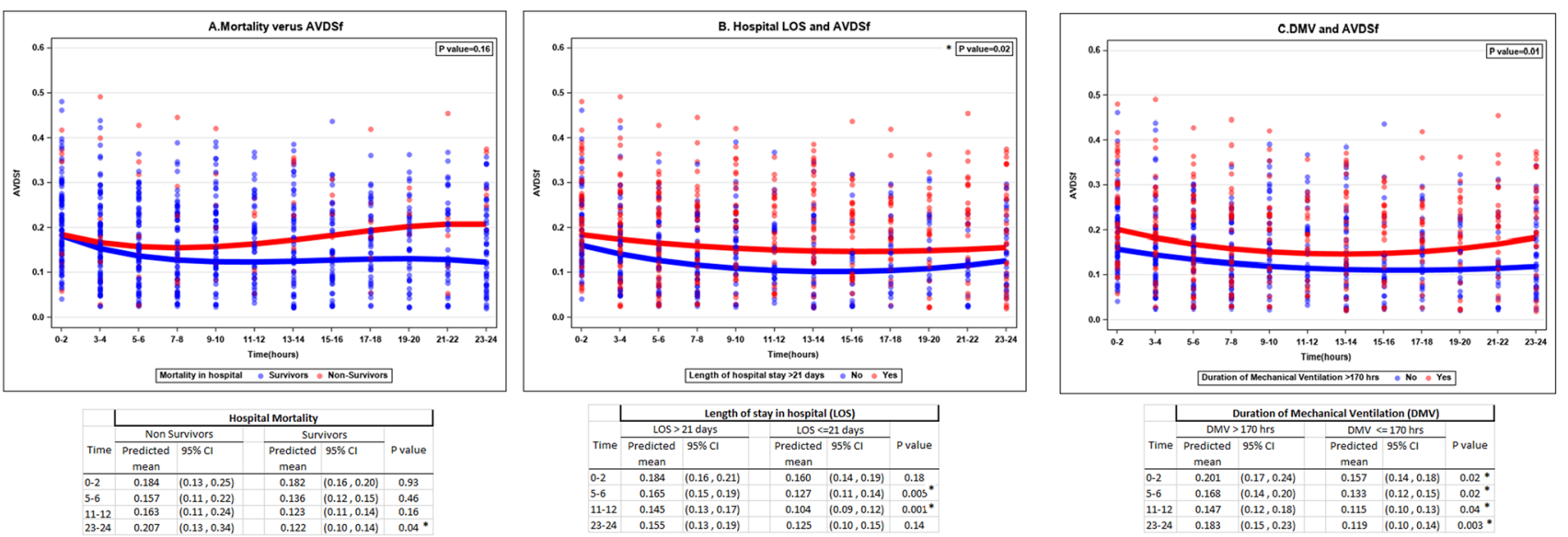

Fig. 2 AVDSf scatter trend plots with time intervals for A) mortality, B) hospital LOS, and C) DMV. * $p$ value $<0.05$

Table 2 Odds ratio comparison for clinical outcomes at 23-24 h when AVDSf $>0.25$. An AVDSf value $>0.25$ was associated with increased odds of mortality by 4.9

\begin{tabular}{llll}
\hline Outcomes & Odds ratio & $95 \%$ CI & $P$-value \\
\hline Mortality & 4.90 & $1.45-16.60$ & 0.0016 \\
DMV & 2.06 & $1.14-3.71$ & 0.0091 \\
Hospital LOS & 1.43 & $0.84-2.45$ & 0.184 \\
\hline
\end{tabular}

$C I$ Confidence Interval, $D M V$ duration of invasive Mechanical ventilation, $L O S$ length of stay

period, we report that AVDSf measured at bedside, in a simple non-invasive manner, may be used to predict postoperative outcomes, including DMV, hospital LOS, and mortality. These findings are similar to other previously published studies in which pulmonary dead space fraction was observed to be a predictor of hospital outcomes [5, 20]. This study further adds to the current limited data with regards to the use of AVDSf in the postoperative period after repair of CHD on CPB, as a valuable tool to predict postoperative outcomes in this high-risk patient population.
In this study, we found that higher AVDSf at 23-24 h interval is associated with hospital mortality and prolonged DMV in postoperative patients with CHD and performed better than other commonly used surrogates of $\mathrm{CO}$ (Fig. 6A-C). Our result is consistent with Yehya et al. [20], who reported that AVDSf $>0.31$ in pediatric patients diagnosed with acute respiratory distress syndrome is associated with mortality, with an area under the ROC of 0.76 (95\% CI, 0.66-0.85; $\mathrm{p}<0.001$ ). In their retrospective study, they found that AVDSf performed better than either initial oxygenation index or $\mathrm{PaO}_{2} / \mathrm{FiO}_{2}$. Similarly, our findings are consistent with those of Ong et al. [11] who found that patients with high dead space following cardiac surgery, had prolonged DMV and hospital LOS.

In contrast to our results, Shakti et al. [5] reported that in patients with single ventricle who underwent stage 1 palliation, higher $\mathrm{Vd} / \mathrm{Vt}$ during the first 48 postoperative hours was associated with longer DMV and hospital length of stay; however, our subgroup analysis for both patient with both intracardiac shunts and single ventricle physiology populations did not demonstrate a significant association between elevated AVDSf and mortality, hospital LOS, or
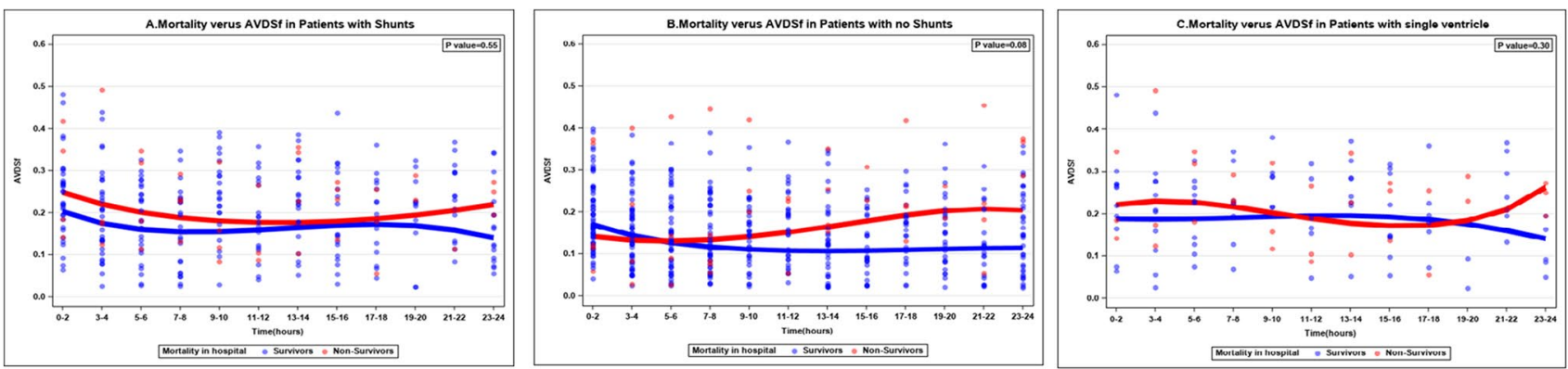

Fig. 3 AVDSf scatter trend plots with time intervals for mortality in patients with A) two ventricles and intracardiac shunts, B) two ventricles without intracardiac shunts, and $\mathbf{C}$ ) single ventricle physiology. ${ }^{*} p$ value $<0.05$ 

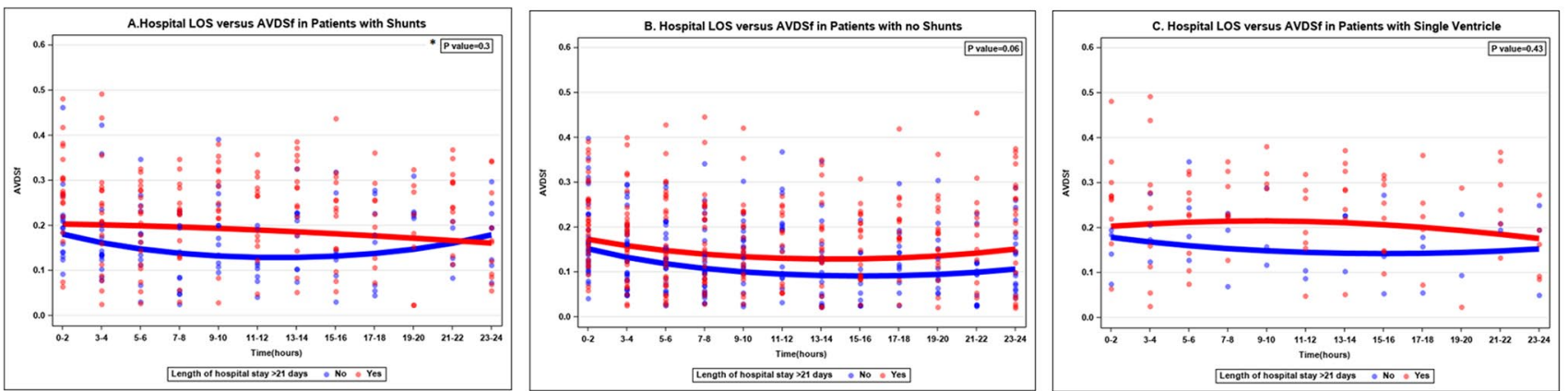

Fig. 4 AVDSf scatter trend plots with time intervals for hospital LOS in patients with A) two ventricles and intracardiac shunts, B) two ventricles without, and $\mathbf{C}$ ) single ventricle physiology. $* p$ value $<0.05$
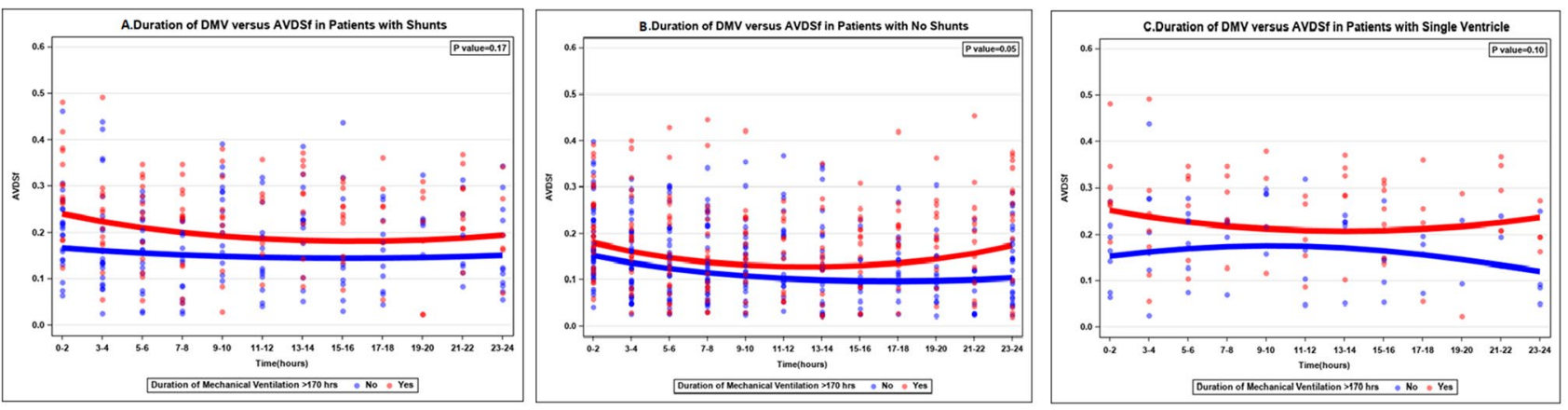

Fig. 5 AVDSf scatter trend plots with time intervals for DMV in patients with A) two ventricles and intracardiac shunts, B) two ventricles without, and $\mathbf{C}$ ) single ventricle physiology. ${ }^{*} p$ value $<0.05$

DMV. This could be explained by the fact that mechanisms behind increased dead space in patients with single ventricle patients during the postoperative period are multiple and not only the result of the inflammatory response to CPB, LCOS, or microvascular thrombosis, but could be secondary to decreased pulmonary blood flow associated with shunts placement or alveolar overdistention secondary to the use of higher tidal volumes in this population [21].

It is important to understand that the presence of shunt increases not only the alveolar-arterial $\mathrm{O}_{2}$ difference but also the arterial-alveolar $\mathrm{CO}_{2}$ difference and, therefore, increases calculated physiological dead space [22]. It is established that a shunt-mediated contribution to the alveolar dead space calculation would be increased by either a decrease in cardiac output or increase in metabolic rate, where either change would increase the mixed venous $\mathrm{PCO}_{2}$; however, a presence of large shunt is required to achieve such results. It should also be noted that in the presence of a right-to-left shunt the Enghoff modification of the Bohr equation overestimates physiological dead space due to venous admixture [23, 24].

Our study results were consistent with the above findings, since AVDSf in our study failed to perform as well, in patient sub-groups with residual shunts and single ventricle physiology (Figs. 3, 4, 5). On contrary, despite some of these limitations, several recent studies from Shostak et al. [17], Koth et al. [25], and Shakti et al. [6] have demonstrated the effective role of elevation of AVDSf on poor postoperative outcomes, including prolonged mechanical ventilation and longer ICU length of stay specifically in the immediate postoperative period. All these studies were largely comprised with patients having an underlying shunt physiology and/ or single ventricle physiology patients. These studies did recognize the likelihood of overestimating $\mathrm{Vd} / \mathrm{Vt}$ in patients with single ventricle and those with residual shunt physiology, similarly the lack of exact etiology for elevation of dead space or shunt fraction calculation is also a limitation of our study.

To have a more detailed pictures, we also report the correlation between the primary outcomes and some surrogates of cardiac output. We found that patients who had higher lactate levels, lower $\mathrm{pH}$, and lower renal $\mathrm{rSO}_{2}$ had longer hospital LOS, and patients who had lower $\mathrm{pH}$ had longer DMV. However, none of these surrogates had any impact on the patient's outcomes with regard to our primary outcome (mortality). 


\begin{tabular}{|c|c|c|c|}
\hline Variable & AUC & Standard error & $95 \%$ CI \\
\hline AVDSf & 0.889 & 0.114 & $0.665-1.000$ \\
\hline Cerebral rSO2 & 0.393 & 0.293 & $0.000-0.966$ \\
\hline Renal rSO2 & 0.543 & 0.176 & $0.198-0.888$ \\
\hline Lactate & 0.778 & 0.11 & $0.561-0.995$ \\
\hline MAP & 0.564 & 0.287 & $0.0008-1.000$ \\
\hline PH & 0.521 & 0.220 & $0.089-0.953$ \\
\hline
\end{tabular}
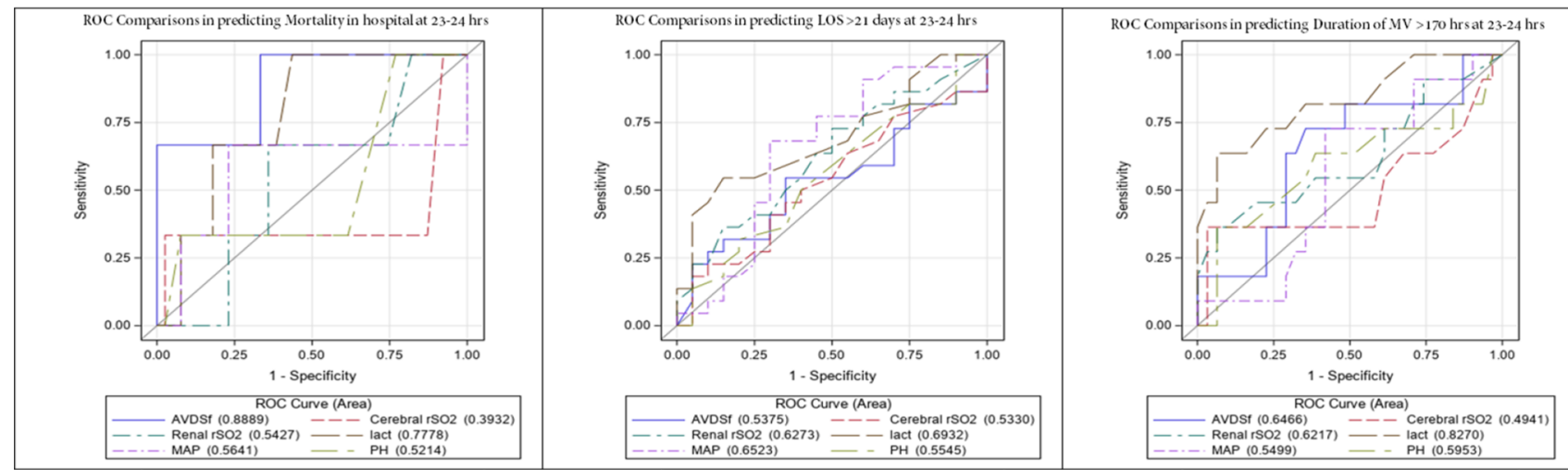

Fig. 6 Receiver operation for AVDSf, arterial blood gases, serum lactate, mean arterial blood pressure, cerebral and renal $\mathrm{rSO}_{2}$ at 23-24 h postoperatively to predict mortality, B) hospital LOS, and C) DMV. AUC and 95\% confidence intervals (CI) for mortality ROCs are given

This report has significant limitations. First, it's a single-center, retrospective study. Second, the findings highlighted in this study might not be generalizable to other institutions or patient populations and therefore, should be validated in adequately powered prospective studies. Third, the calculation of the AVDSf may have been affected by the retrospective data collection of the $\mathrm{EtCO}_{2}$ values, which were not charted concurrently with the $\mathrm{PaCO}_{2}$ values at times. Finally, our study was not able to determine the exact underlying etiology for elevation of alveolar dead space fractions.

Although our findings cannot support any conclusions regarding postoperative management of critically ill children after cardiac surgery, it emphasizes a further need for more future prospective, randomized, and adequately powered interventional trials to determine whether early interventions based on input from capnography could improve clinical outcomes and reduce both DMV and mortality in these high-risk groups.

\section{Conclusion}

In this retrospective study of children having surgery requiring CPB for CHD, we found that higher AVDSf values in the immediate postoperative period are associated with mortality, DMV, and hospital LOS.

Supplementary Information The online version contains supplementary material available at https://doi.org/10.1007/s00246-021-02674-2.

Authors' Contributions IS participated in the conceptualization, methodology, data curation, and original draft preparation. SH participated in the conceptualization, methodology, and reviewing and editing of the manuscript. VR participated in the methodology, data analysis, and 
reviewing and editing of the manuscript. PA participated in the reviewing and editing of the manuscript. ME participated in the reviewing and editing of the manuscript. AA participated in the conceptualization, methodology, data curation, original draft preparation, and reviewing and editing of the manuscript.

\section{Funding Not applicable}

Data Availability The datasets used and analyzed during the current study are available from the corresponding author on reasonable request.

\section{Declarations}

Conflict of interest Dr. Eldridge received salary support for research from the National Heart, Lung, and Blood Institute (NHLBI) of the National Institutes of Health (R01 HL115061-04 and R01HL105820) and the U.S. Department of Defense, Navy (N00024-17-C-4318 and N0463A-12-C-0004). The remaining authors have disclosed that they do not have any potential conflicts of interest.

Ethical Approval The Institutional Review Board (IRB) at the University of Wisconsin, Madison approved the study protocol with a waiver of informed consent.

Consent for Publications Not applicable.

\section{References}

1. Wessel DL (2001) Managing low cardiac output syndrome after congenital heart surgery. Crit Care Med 29(10 Suppl):S220-S230

2. Cigarroa CL, van den Bosch SJ, Tang X, Gauvreau K, Baird CW, DiNardo JA et al (2018) Measurement of dead space fraction upon ICU admission predicts length of stay and clinical outcomes following bidirectional cavopulmonary anastomosis. Pediatr Crit Care Med 19(1):23-31

3. Lyew MA, Bacon DR, Nesarajah MS (1996) Right ventricular perforation by a pulmonary artery catheter during coronary artery bypass surgery. Anesth Analg 82(5):1089-1090

4. Booth KL, Mercer-Smith G, McConkey C, Parissis H (2012) Catheter-induced pulmonary artery rupture: haemodynamic compromise necessitates surgical repair. Interact Cardiovasc Thorac Surg 15(3):531-533

5. Shakti D, McElhinney DB, Gauvreau K, Yarlagadda VV, Laussen PC, Betit P et al (2014) Pulmonary deadspace and postoperative outcomes in neonates undergoing stage 1 palliation operation for single ventricle heart disease. Pediatr Crit Care Med 15(8):728-734

6. West JB (1995) Ventilation. In: Coryell PA (ed) Respiratory Physiology-The essentials, 5th edn. Williams \& Wilkins, Baltimore, pp $11-20$

7. Zhang YJ, Gao XJ, Li ZB, Wang ZY, Feng QS, Yin CF et al (2016) Comparison of the pulmonary dead-space fraction derived from ventilator volumetric capnography and a validated equation in the survival prediction of patients with acute respiratory distress syndrome. Chin J Traumatol 19(3):141-145

8. Cepkova M, Kapur V, Ren X, Quinn T, Zhuo H, Foster E et al (2007) Pulmonary dead space fraction and pulmonary artery systolic pressure as early predictors of clinical outcome in acute lung injury. Chest 132(3):836-842

9. Lucangelo U, Bernabe F, Vatua S, Degrassi G, Villagra A, Fernandez R et al (2008) Prognostic value of different dead space indices in mechanically ventilated patients with acute lung injury and ARDS. Chest 133(1):62-71

10. West JB (2014) Respiratory physiology: the essentials. Wolters Kluwer/Lippincott Williams \& Wilkins, Philadelphia, Pennsylvania

11. Ong T, Stuart-Killion RB, Daniel BM, Presnell LB, Zhuo H, Matthay MA et al (2009) Higher pulmonary dead space may predict prolonged mechanical ventilation after cardiac surgery. Pediatr Pulmonol 44(5):457-463

12. Beitler JR, Thompson BT, Matthay MA, Talmor D, Liu KD, Zhuo $\mathrm{H}$ et al (2015) Estimating dead-space fraction for secondary analyses of acute respiratory distress syndrome clinical trials. Crit Care Med 43(5):1026-1035

13. Frankenfield DC, Alam S, Bekteshi E, Vender RL (2010) Predicting dead space ventilation in critically ill patients using clinically available data. Crit Care Med 38(1):288-291

14. Siddiki H, Kojicic M, Li G, Yilmaz M, Thompson TB, Hubmayr $\mathrm{RD}$ et al (2010) Bedside quantification of dead-space fraction using routine clinical data in patients with acute lung injury: secondary analysis of two prospective trials. Crit Care 14(4):R141

15. Hardman JG, Aitkenhead AR (2003) Estimating alveolar dead space from the arterial to end-tidal $\mathrm{CO}(2)$ gradient: a modeling analysis. Anesth Analg 97(6):1846-1851

16. Bhalla AK, Rubin S, Newth CJ, Ross P, Morzov R, Soto-Campos $\mathrm{G}$ et al (2015) Monitoring dead space in mechanically ventilated children: volumetric capnography versus time-based capnography. Respir Care 60(11):1548-1555

17. Shostak E, Schiller O, Merzbach A, Shochat T, Amir G, Frenkel G et al (2020) Alveolar dead-space fraction and arterial saturation predict postoperative course in fontan patients. Pediatr Crit Care Med 21(4):e200-e206

18. Cepkova M, Kapur V, Ren X et al (2007) Pulmonary dead space fraction and pulmonary artery systolic pressure as early predictors of clinical outcome in acute lung injury. Chest 132:836-842

19. Ghuman AK, Newth CJ, Khemani RG (2012) The association between the end tidal alveolar dead space fraction and mortality in pediatric acute hypoxemic respiratory failure. Pediatr Crit Care Med 13(1):11-15

20. Yehya N, Bhalla AK, Thomas NJ, Khemani RG (2016) Alveolar dead space fraction discriminates mortality in pediatric acute respiratory distress syndrome. Pediatr Crit Care Med 17(2):101-109

21. Bhalla A, Markovitz BP (2014) Neonatal single-ventricle palliative surgery: lost in (dead) space? Pediatr Crit Care Med 15(8):777-778

22. Robertson HT (2015) Dead space: the physiology of wasted ventilation. Eur Respir J 45(6):1704-1716

23. Burrows FA (1989) Physiologic dead space, venous admixture, and the arterial to end-tidal carbon dioxide difference in infants and children undergoing cardiac surgery. Anesthesiology 70(2):219-225

24. Suarez-Sipmann F, Santos A, Böhm SH, Borges JB, Hedenstierna G, Tusman G (2013) Corrections of Enghoff's dead space formula for shunt effects still overestimate Bohr's dead space. Respir Physiol Neurobiol. 189(1):99-105

25. Koth AM, Kwiatkowski DM, Lim TR, Bauser-Heaton H, Asija R, McElhinney DB, Hanley FL, Krawczeski CD (2018) Association of dead space ventilation and prolonged ventilation after repair of tetralogy of Fallot with pulmonary atresia. J Thorac Cardiovasc Surg 156(3):1181-1187

Publisher's Note Springer Nature remains neutral with regard to jurisdictional claims in published maps and institutional affiliations. 